\title{
Systematising Pharmacovigilance Engagement of Patients, Healthcare Professionals and Regulators: A Practical Decision Guide Derived from the International Risk Governance Framework for Engagement Events and Discourse
}

\author{
Priya Bahri ${ }^{1} \cdot$ Antoine Pariente $^{2}$
}

Accepted: 5 August 2021 / Published online: 15 September 2021

(c) The Author(s) 2021

\begin{abstract}
Introduction Input from patients and healthcare professionals to regulatory assessments is essential for benefit-risk management of medicines. How to best obtain input in different risk scenarios is uncertain.

Objectives The objective of this study was to investigate whether the International Risk Governance Council (IRGC) Framework is applicable to pharmacovigilance and can guide selecting engagement mechanisms for optimising stakeholder input. Methods For proof-of-concept, classify 'iconic' cases of pharmacovigilance engagement at the European Medicines Agency (EMA) by IRGC risk scenario types and compare the engagement that happened with the engagement discourse recommended by the IRGC Framework for different risk scenarios. If the concept is proven, derive proposals for strengthening engagement.

Results Six iconic cases were classified by risk scenario type at the respective time points when deciding on engagement: venous thromboembolism with combined hormonal contraceptives (complex risk); lipodystrophy with highly active antiretroviral therapy medicines, carcinogenicity with contaminated nelfinavir products (uncertain risks); teratogenicity with thalidomide, progressive multifocal leukoencephalopathy with natalizumab, teratogenicity and developmental disorders with valproate (ambiguous risks). The comparison of the engagement events with IRGC recommendations showed correspondence between the scope/outcomes of the events and the features of the recommended discourse.

Conclusions The IRGC Framework appears applicable to pharmacovigilance. Proposals derived from the IRGC recommendations may be valuable for guiding regulators when selecting mechanisms for engagement with patients and healthcare professionals in given risk scenarios. The proposed decision guide aims at ensuring systematic and consistent engagement across regulatory assessments and providing for the most purposeful discourse, to effectively obtain real-world input for regulatory risk assessment, evaluation of risk minimisation measures and decision making.
\end{abstract}

\section{Introduction}

Collaboration of all stakeholders involved in developing or using medicines, and input specifically from patients and healthcare professionals, are considered essential for effective pharmacovigilance and benefit-risk management

Priya Bahri

priya.bahri@ema.europa.eu

1 Pharmacovigilance Office, European Medicines Agency (EMA), Domenico Scarlattilaan 6, 1083 HS Amsterdam, The Netherlands

2 Team Pharmacoepidemiology, Bordeaux University, INSERM, U1219 BPH Research Center, Bordeaux, France of medicines [1-3]. Such input may give insights into the use of medicines, the meaning of risks in healthcare and patient life, the occurrence of adverse reactions and its circumstances, as well as the impact of risks, risk communication and measures for risk management, all with the view to understand and improve patient safety. Therefore, regulatory bodies nowadays solicit more often, and more comprehensively, real-world input from patient and healthcare professional representatives to support risk assessments and decision making on granting and maintaining marketing authorisations of medicinal products, and to inform related regulatory policies. Product-related decisions in the postauthorisation phase relate mostly to safe use advice, restrictions of use and other specific risk minimisation measures; 


\section{Key Points}

Points to consider for engagement of regulators, patients and healthcare professionals are under development at the European Medicines Agency (EMA) in order to ensure systematic, consistent and effective engagement for real-world input to risk assessments, evaluation of risk minimisation measures and decision making.

The analysis of six cases of major safety concerns for which different engagement mechanisms were used by EMA for the first time shows that the framework of the International Risk Governance Council (IRGC) appears applicable to pharmacovigilance activities.

A decision guide that brings together the IRGC recommendations and the regulatory risk assessment process, to support selecting discourse types and engagement mechanisms in given risk scenarios, is proposed for piloting at EMA and refinement as a tool for regulators.

other options include further investigations, keeping a safety concern under close monitoring, precautionary measures, suspension of marketing or withdrawal of the marketing authorisation. A risk assessment may also conclude that no action is necessary.

\subsection{Concept of Pharmacovigilance Engagement}

Stakeholder engagement for pharmacovigilance purposes has recently been conceptualised as an ongoing process of knowledge exchange among stakeholders. The term 'engagement' implies the crucially important mutuality of this process. Pharmacovigilance stakeholders include patients and their carers, healthcare professionals, regulatory bodies, other health and healthcare organisations, marketing authorisation holders and industry in general, academia, and the wider public. The process is enacted through engagement events with the steps of preparing, conducting and evaluating such events. Knowledge exchange refers to sharing perspectives, norms, values and meanings as well as scientific and real-world knowledge. Adoption of knowledge is meant to lead to positive changes in understandings, attitudes, behaviours and policies as the main direct outcomes, and ultimately to lead to positive health outcomes. Conceptually, engagement is seen as having multiple dimensions, both at process and outcome level [4] (see Table 1).

\subsection{Mechanisms for Regulatory Engagement at the European Medicines Agency}

In the European Union (EU), the European Medicines Agency (EMA) has, since its establishment in 1995, increasingly responded to patients' and healthcare professionals' calls for involvement, taken initiatives to drive mutual engagement further, and thus over time established different engagement mechanisms. New legislation in 2012 replaced the Pharmacovigilance Working Party of EMA's Committee for Medicinal Products for Human Use (CHMP) with the Pharmacovigilance Risk Assessment Committee (PRAC), which has members coming not only from the competent authorities of each EU Member State but also from the patient and healthcare professional communities. In addition, PRAC has made use of all available mechanisms for engagement events to support their assessments (see Table 2). Between 2015 and 2019, PRAC initiated 130 engagement events for 71 medicinal products. The majority of these events were written consultations, mostly for the review of safety communications, while 22 events involved deeper forms of engagement with in-person, face-to-face interactions. PRAC is also interested in the involvement of patients and healthcare professionals in planning and evaluating risk minimisation measures to support the safe use of medicines in healthcare. This interest stems from PRAC's experience that current evaluations of these measures, legally imposed and provided by marketing authorisation holders as post-authorisation safety studies, do not always demonstrate effectiveness in terms of achieving the defined risk minimisation objectives or that the methods evaluating effectiveness are not always robust. Current evaluations may also not reveal why measures are not effective.

Questions about how to best obtain stakeholder input for different safety concerns and for different kinds of input needed during risk assessments have been raised by PRAC in the context of its 'Strategy for Measuring the Impact of Pharmacovigilance Activities' [6]. Especially challenging

Table 1 Dimensions of stakeholder engagement for pharmacovigilance purposes [4]

\begin{tabular}{ll}
\hline Dimension & Description \\
\hline Breadth & Quantity and diversity of stakeholders \\
Depth & Extent of knowledge shared (in order of increasing depth: information, consultation and participation [5]) \\
Texture & $\begin{array}{c}\text { Interactive dynamics of what the engagement feels like, what it means to people, and how this shapes } \\
\text { motivations to engage and change behaviours based on values, emotions, (mis)trust and rationales }\end{array}$ \\
\hline
\end{tabular}


are safety concerns that carry uncertainty or controversy regarding the evidence on the risk and the impact of possible regulatory actions. Such questions are also discussed in the US [7], where a series of guidance from the US Food and Drug Administration on enhanced incorporation of the 'patient's voice' in medicines development and regulatory decisions are currently being drawn up [8]. While the US FDA holds a public hearing for each advisory committee meeting [9], PRAC has so far convened only two public hearings. The option of a public hearing was added for certain regulatory procedures by EU legislation that came into force in 2012. High-level criteria are applied by PRAC when deciding to call for a hearing or choose another engagement mechanism instead. These criteria are feasibility to hold a public hearing in light of the urgency of the matter; nature and extent of the safety concern; therapeutic effect of the medicine and availability of therapeutic alternatives; potential impact of regulatory actions on therapeutic practice and availability of treatments; and level of public interest [10]. The criterion 'nature and extent of the safety concern' has so far not been further detailed beyond the seriousness and frequency of the identified or potential adverse reaction; this constitutes an area of uncertainty regarding deciding on how to arrange for systematic, consistent and effective engagement.

\subsection{International Risk Governance Framework}

In general, safety concerns arise from nature as well as technologies, not only in the area of medicine but linked, for example, to energy supply, food production, waste management, environmental and climate change or data security. Many civil society movements and organisations have started or contributed to the debates, and platforms for their interaction with policymakers have been created. It is hence of interest to see if experiences with stakeholder engagement in other risk areas may provide learnings for pharmacovigilance. The International Risk Governance Council (IRGC) has established a practical engagement framework that builds on the experience from various risk areas. It integrates characteristics of typical risk scenarios with evidencebased recommendations for risk management strategies and discourse within engagement of affected populations, experts and other stakeholders $[12,13]$ (see Table 3 ). This framework has not yet been applied for pharmacovigilance purposes; however, its features fit well with the objectives and current challenges of pharmacovigilance. Namely, the framework supports a multidisciplinary and multistakeholder approach to risk, aiming to provide and structure scientific evidence within the societal context for risk management and social benefit. It further aims to guide coping with risks in situations of high complexity, uncertainty or ambiguity, based on principles of transparency, strategic focus, efficiency, accountability, sustainability, equity, compliance with the law, and acceptability from the viewpoints of ethics, the public and policymaking [12].

\section{Objectives}

As there is uncertainty about which engagement mechanism may be best in given typical scenarios of safety concerns with medicines in terms of the nature and extent of the concern, this study investigated whether the IRGC Framework $[12,13]$ is applicable to regulatory pharmacovigilance and can possibly guide regulators when selecting mechanisms of stakeholder engagement during risk assessment procedures.

Overall, this aims at optimising engagement by ensuring that it is systematic and consistent across regulatory procedures and obtains, most effectively, real-world input from patients and healthcare professionals for regulatory risk assessment, evaluation of risk minimisation measures and decision making in different risk scenarios. The results of this study are meant to inform ongoing work of the PRAC Interest Group on Measuring the Impact of Pharmacovigilance Activities, in particular the development of points to consider supporting PRAC in enhancing their engagement with patients and healthcare professionals.

Table 2 Mechanisms for stakeholder engagement events available to the Pharmacovigilance Risk Assessment Committee at the European Medicines Agency[11]

\section{Stakeholder mechanism}

Written consultations (public and open to all citizens and organisations as announced on the EMA website, e.g. public consultations on guidelines and other policy documents, and/or announced to organisations of the EU stakeholder network, e.g. targeted reviews of planned safety communication documents)

Dedicated meetings (non-public meetings with stakeholder representatives, in particular patients and healthcare professionals, e.g. so-called ad hoc meetings and research oversight meetings)

Participation of additional scientific/clinical experts and/or patient representatives in a meeting of the Scientific Advisory Groups established for various therapeutic areas (non-public meetings)

Public hearings (open to all citizens and organisations as announced on the EMA website and provided to the public as life-broadcast)

EMA European Medicines Agency, EU European Union 


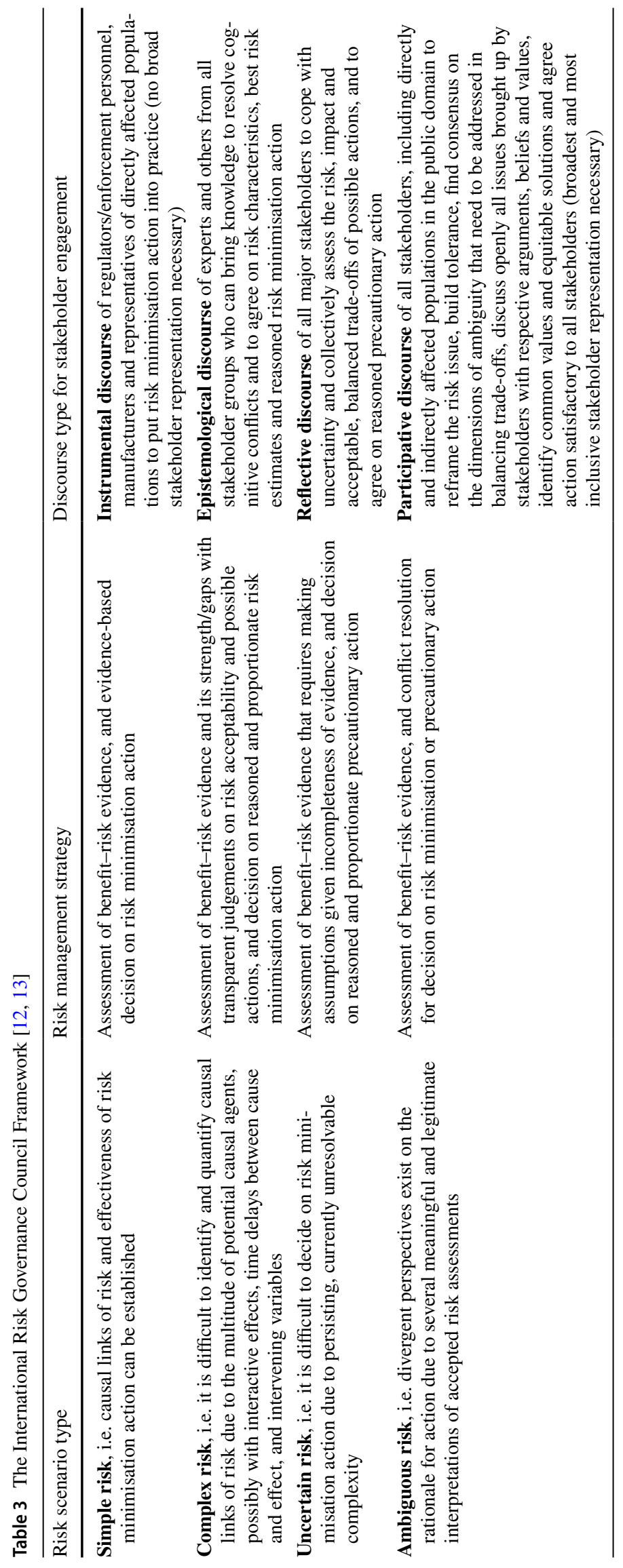




\section{Methods}

The applicability of the IRGC Framework to pharmacovigilance was investigated as a proof-of-concept study by applying the framework to cases of safety concerns with medicinal products for which different mechanisms for engagement with patients and healthcare professionals were used at EMA for the first time during a risk assessment procedure (see Table 4). In this article, these cases are referred to as 'iconic' in the meaning of 'distinctive', 'symbolic', 'impressive' and 'widely recognised' [14-16], as these cases are well-known and frequently referred to in the pharmacovigilance as well as patient advocacy communities. Cases of the first-time use of written consultations on draft wording of product information, medicines safety advisories on the EMA website or direct healthcare professional communications (DHPCs) were excluded in order to focus on deeper (and still rarer) engagement with face-to-face interaction, including with those who have been harmed by medicines.

The applicability analysis comprised for each iconic case: (1) classifying the safety concern as it was understood at the time point of deciding on engagement according to the IRGC risk scenario typology (see Table 3); and (2) characterising the engagement event that happened in terms of mechanism (see Table 2), scope and the dimensions of breadth, depth and texture (see Table 1), and comparing it with the engagement discourse recommended by the IRGC Framework for the different risk scenarios (see Table 3).

The analysis used regulatory documentation published by EMA and published case studies as referenced, as well as some internal EMA working documents to cross-check details of the arrangements for the engagement events.

The analytical approach was consulted with the PRAC Interest Group on Measuring the Impact of Pharmacovigilance Activities, which includes patient and healthcare professional representatives. The case studies used for the analysis also involved patient views, interviews and surveys.

The potential for researcher bias in data interpretation was minimised by a structured approach to data analysis, inclusion of results from published case studies and a review of the analysis from the first author by the second author.

As the concept was proven, proposals were derived from the IRGC Framework for guiding regulators when selecting engagement mechanisms for different risk scenarios. Had the proof-of-concept analysis led to a negative result, further investigations on the specifics of risks with medicines and different needs for risk governance would have been warranted.

\section{Results of the Proof-of-Concept Study}

The risk scenario classification step of analysing the iconic cases showed that none of the risks for which the various engagement mechanisms were used for the first time at EMA could be classified as a simple risk. One case, i.e. combined hormonal contraceptives (CHCs), was classified as a complex risk; two cases, i.e. medicines used for highly active antiretroviral therapy (HAART) and nelfinavir-containing products, were classified as uncertain risks at the time of initiating engagement; and three cases, i.e. thalidomide, natalizumab and valproate, were classified as ambiguous risks.

The next step of the analysis, i.e. comparing the engagement mechanisms used in the iconic cases with the engagement discourse types recommended by the IRGC Framework for the various risk scenarios, showed the following: EU regulators, while not aware of the IRGC Framework, decided on engagement that was consistent with the IRGC recommendations. For the complex risk of CHCs, the scope of the dedicated meeting and its outcome to present risk estimates

Table 4 Iconic cases of pharmacovigilance engagement at the European Medicines Agency, in chronological order

Iconic case of pharmacovigilance engagement

Risk of lipodystrophy with medicines used for highly active antiretroviral therapy: First-time engagement of patient and healthcare professional representatives in a multistakeholder oversight committee for research requested by EMA for an adverse reaction suspected and notified by patients themselves (1999)

Risk of potential carcinogenicity with contaminated nelfinavir-containing products: First-time engagement of EMA where a patient representative was contacted by EMA immediately after a marketing authorisation holder's notification of a quality defect and before the risk assessment could be started. [Note: The risk assessment demonstrated that the exposure of patients had been below the toxic threshold] (2007)

Risk of teratogenicity with thalidomide: First-time engagement of EMA where victim and patient representatives were brought together at a dedicated meeting (2007)

Risk of progressive multifocal leukoencephalopathy with natalizumab: First-time invitation of patient representatives in a Scientific Advisory Group meeting at EMA regarding the risk of an authorised medicine (2008)

Risk of venous thromboembolism with combined hormonal contraceptives: First-time dedicated meeting with patient and healthcare professional representatives for EMA's Pharmacovigilance Risk Assessment Committee established in July 2012 under then new legislation (2013)

Risk of teratogenicity with valproate: First-time public hearing at EMA's Pharmacovigilance Risk Assessment Committee (PRAC) (2017)

EMA European Medicines Agency 
in the product information for patients and healthcare professionals differently can be seen as corresponding with the purpose of an epistemological discourse to resolve cognitive conflicts between stakeholders regarding risk characteristics, estimates and risk minimisation action. For the, at the time, uncertain risk of HAART products, the engagement scope of multistakeholder research oversight corresponds with the purpose of a reflective discourse to collectively assess risk. For nelfinavir-containing products, the engagement scope included motivating patients to seek appropriate treatment management. This can be interpreted as an engagement discourse with reflection on the trade-offs between the needs for further risk assessment and safeguarding patient health during investigations and precautionary product suspension. For the ambiguous features of the risks of thalidomide, natalizumab and valproate, the engagement events can all be analysed as corresponding with a participative discourse. In all these three cases, considerations that patients should not be deprived from necessary and beneficial treatments, provided that risk minimisation measures can be agreed that are effective and satisfactory to all stakeholders affected differently, were at the core of engagement (see Table 5).

The results of the analysis can be taken as proof that the IRGC Framework appears applicable to pharmacovigilance and that the IRGC recommendations may be valuable for guiding regulatory pharmacovigilance engagement.

\section{Discussion}

This proof-of-concept study demonstrated that the IRGC Framework, which is well-established for stakeholder engagement in various risk areas of nature and technology, also appears applicable to pharmacovigilance, as EU regulators, albeit not consciously, engaged with stakeholders in iconic cases of safety concerns with medicines over the past two decades in ways that correspond remarkably with the nature of engagement that would have emerged if the IRGC Framework had been applied.

We can only speculate why the IRGC Framework has not been applied for pharmacovigilance purposes yet. Maybe the comprehensive regulation of medicines has long been viewed as a sufficient framework for risk governance. At the origin of this regulation more than 50 years ago, patients have likely been seen, only in a traditional perspective, as vulnerable and to be cared for. However, their active role in regulatory activities has been steadily increasing, at the latest since the patient advocacy for anti-human immunodeficiency virus (HIV) treatments in the 1980s [24, 25]. This study confirms that EMA, almost immediately after becoming operational in 1995, embraced, welcomed and encouraged the active role of patient and healthcare professional representatives in regulatory pharmacovigilance activities.
The iconic cases that were analysed in this study occurred between 1999 and 2017, and their crucial importance for gradually building up more participation and trust of stakeholders in the EU regulatory pharmacovigilance system can, from an EMA perspective, not be underestimated. The trust in EMA's high safety standards was also reiterated recently by stakeholders at a public EMA meeting on vaccines against COVID-19, the disease caused by the severe acute respiratory syndrome coronavirus 2 (SARS-CoV-2) [36]. The importance of the iconic cases justifies their analysis to learn for the future.

Uncertainty has been expressed by PRAC about optimal stakeholder engagement, together with their intent to enhance engagement in systematic and consistent manner across all regulatory risk assessments as part of the PRAC Impact Strategy [6]. Hence, considering the recommendations of the IRGC Framework may be valuable for guiding pharmacovigilance engagement when selecting engagement mechanisms for effectively obtaining input from patients and healthcare professionals for regulatory risk assessments and decision making. For developing this further on the basis of established approaches to regulatory risk assessment, the following is raised for discussion here.

\subsection{Classification of Safety Concerns with Medicines According to the International Risk Governance Council (IRGC) Risk Scenario Typology}

Although real risks often present as a combination of risk types [12], most of the safety concerns of the iconic cases could be clearly classified according to the IRGC risk scenario typology. One case was difficult to classify. For natalizumab, the risk classification as an uncertain or ambiguous risk was considered in favour of ambiguous risk; first, because the IRGC Framework views uncertainty as intrinsic to human knowledge and thus to any risk assessment [13]. In fact, once medicines have been authorised on the basis of clinical trial data, safety concerns identified in the postauthorisation phase mainly relate to potential adverse reactions that are uncommon rare, occur in patients with specific conditions, or have a delayed onset. Dealing with uncertainty of evidence is therefore intrinsic to pharmacovigilance and risk assessment, from both the clinical diagnosis and the statistical data analysis points of view, and with regard to causality assessment. The second rationale for classifying the natalizumab case as an ambiguous risk was that patient views were crucial for the regulators' decision making, i.e. to either conclude with a negative benefit-risk assessment, or positively with risk acceptance given the value of the treatment benefit for patients. The existence of divergent perspectives was postulated for the risk classification, due to known patient advocacy for maintaining this treatment option on the one hand and regulators' obligations for patient safety 


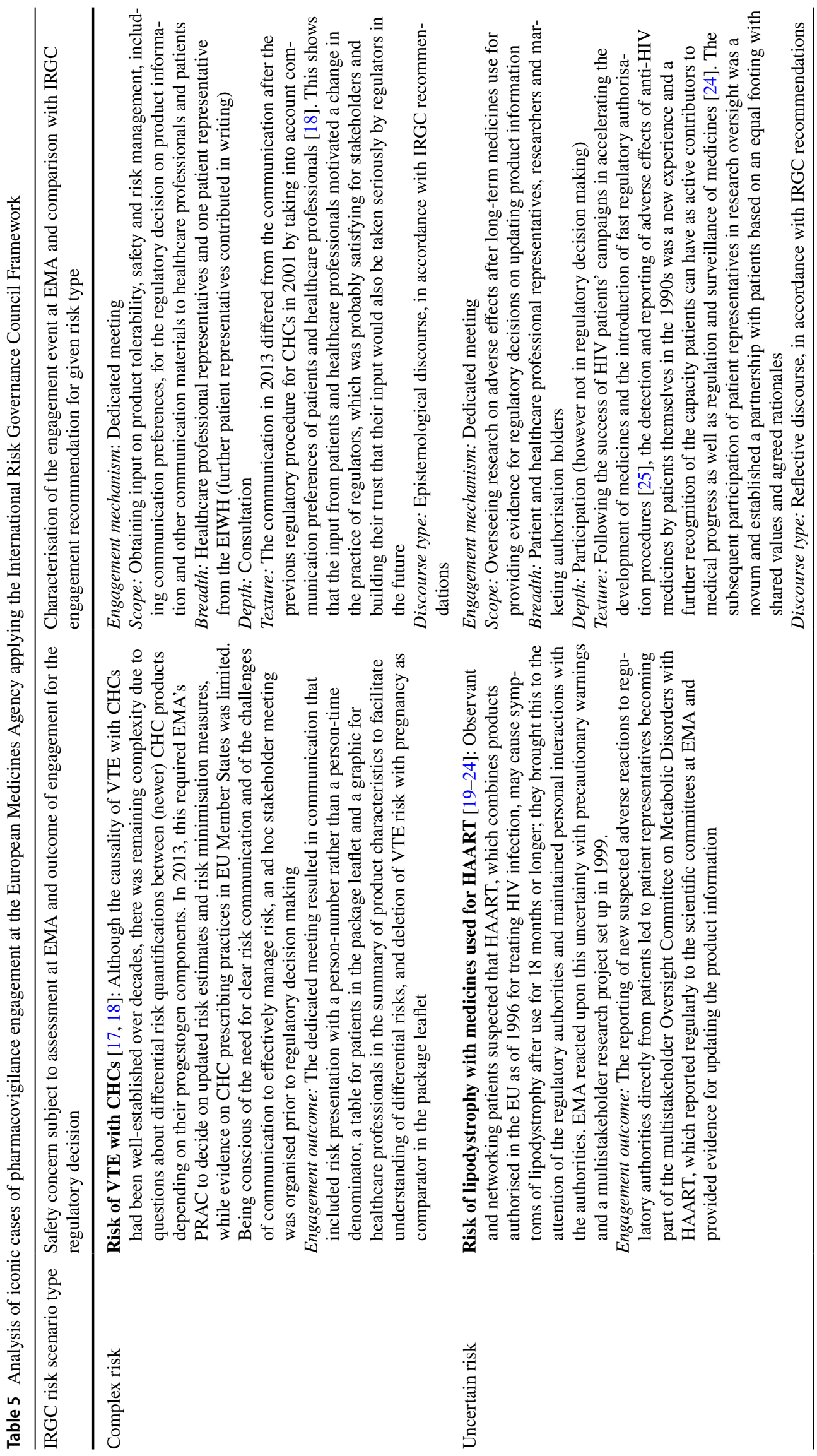




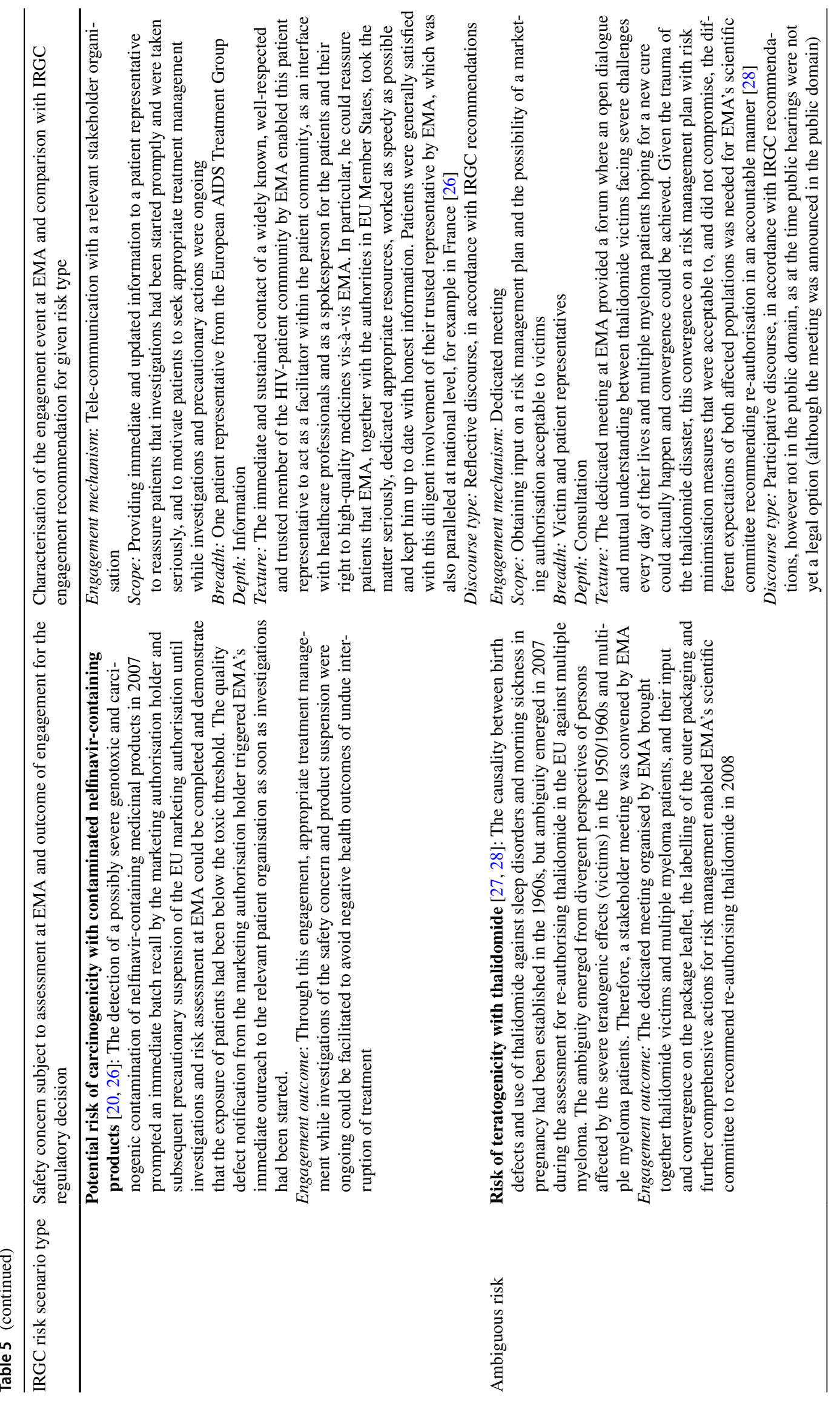




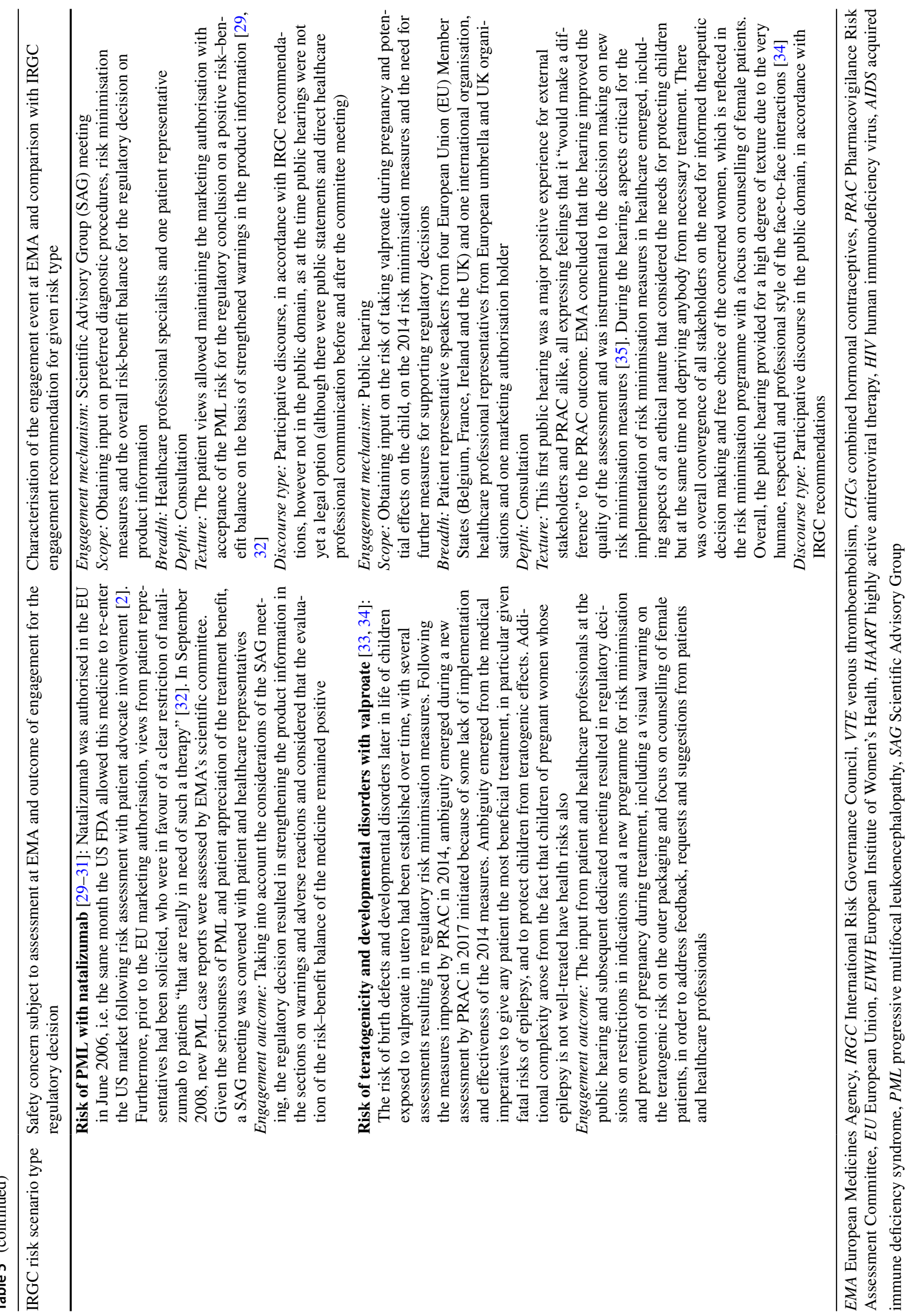


on the other, although there was never a declared conflict between patients and regulators. Actually, in the pre- and post-authorisation phases, both parties had always converged on the need for considering the risk-benefit balance carefully and for potentially identifying suitable patient groups and risk minimisation measures [29-31].

\subsection{Changes in Risk Scenario Type During Regulatory Risk Assessment}

For the analysis of the iconic cases, the risk scenario type was determined as the safety concern was understood before deciding on engagement and finalising the risk assessment. This mimicked the situation when regulators agree timetables for risk assessment procedures, including interactions with stakeholders. However, during risk assessment, the knowledge on the safety concern often changes, and so may the risk scenario: uncertainty usually decreases, but can sometimes also increase when limitations in evidence are identified; complexity often persists; and ambiguity may become apparent only during the assessment or engagement when different stakeholder perspectives are presented. In the case of nelfinavir for example, precautionary action had to be taken urgently, while investigations of the risk, performed as quickly as possible, moved it from an uncertain risk to a complex and even simple risk (eventually, it could be concluded that patient exposure had been below the toxic threshold) [20, 26].

\subsection{Compatibility of IRGC Discourse Recommendations with Medicines Regulation}

Safety concerns with medicines are assessed by PRAC under various regulatory procedures legally prescribed by the source and type of new safety information, the urgency of the safety concern, the kind of medicinal products involved, the authorisation status of these product(s), and sometimes by their link with other procedures ongoing for the product(s). Therefore, the following points require discussion when applying the IRGC discourse recommendations to regulatory procedures for risk assessment:

Availability of Engagement Mechanisms: With the establishment of PRAC under then new legislation in 2012, the PRAC members from the competent authorities in EU Member States were provided with two major novelties for strengthening stakeholder engagement; namely, (1) extension of PRAC membership by one additional member and one alternate member appointed by the European Commission from each of the patient and healthcare professional communities, and (2) the option for PRAC to call for public hearings during specific regulatory procedures, i.e. 'referrals' $[10,37]$. While PRAC's choice for a hearing allowing for a participative discourse in the public domain is restricted to specific regulatory procedures, EMA has also announced dedicated meetings to the public and arranged for wide stakeholder interaction when needed, as in the cases of thalidomide and natalizumab. Furthermore, EMA holds public meetings, albeit not hearings, when major health concerns demand this, just as with the current SARS-CoV-2 pandemic.

Publicity and Patient Privacy of Engagement: Publicity is an important element of patient advocacy for "visibility to the world" (François Houÿez, European Organisation for Rare Diseases [EURORDIS], personal communication, 3 March 2020) and may hence be important to patient representatives in regulatory activities. However, patients also have a right to privacy, and there could be situations where individuals prefer a (participative) discourse with regulators that does not happen in the public domain. PRAC has experienced that finding patient representatives willing to engage can be difficult even for a closed meeting, as happened, for example, when PRAC wanted to explore why risk minimisation measures for preventing pregnancy during treatment with isotretinoin had not been fully effective and how to improve the measures.

Timing of Engagement: As regulatory procedures are subject to legally imposed timetables, stakeholder engagement must be organised in ways that do not delay regulatory action necessary for patient safety. For public hearings, EU legislation even states explicitly that they may be held "where the urgency of the matter permits" [37], which recognises that the orderly conduct of such hearings requires preparation. Notably, many patient and healthcare professional representatives have shown remarkable commitment to respond to the EMA's calls within tight timeframes. Written consultations of urgent communication documents are sometimes finalised even within a few hours.

Availability of Stakeholders: Stakeholder engagement in regulatory activities is pro bono; currently, only travel expenses and daily allowances are provided by EMA to patient and healthcare professional representatives. The representatives may also have to donate their free time off work for travelling and attending EMA meetings. In addition, consultations and meetings are held in English as the EMA working language. These circumstances restrict the availability of representatives and may hinder regulators to hear from all patient, healthcare professional and society segments. In particular, the vast majority of patients are not members of a patient organisation that could make them aware of and prepare them for opportunities of engagement. On the other hand, in some situations even the engagement with a very small breadth at engagement process level can have a wide breadth at engagement outcome level, as shown by the nelfinavir case. In this case, a single patient representative could achieve a high positive impact in terms of treatment management of many patients, as he was an individual 
well-recognised and highly trusted in the patient community [26]. In particular, where regulatory action needs to be taken urgently or within a short time frame, an appropriate breadth of engagement might have to be achieved by finding a few patients and healthcare professionals who can act in intermediary roles or present a full range of perspectives, because interacting with many people might not be feasible due to limited stakeholder availability. However, with a general view, questions about who are the public, in how far it is a coherent entity or how many segments of the public exist, and who can represent the public or patient and healthcare professional communities have to be investigated for further optimising future engagement in regulatory activities [38].

Accountability: EU legislation provides for engagement of stakeholders and transparency [37]. A recent policy analysis reported a high degree of transparency of EMA's risk assessments prescribed by the legislation and EMA policies [39]. While the input from stakeholders to risk assessments is seen by the EU regulatory network as instrumental to good decision making, the ultimate legal responsibility for risk assessments and the terms of marketing authorisations remains with the regulators. Their responsibility for decisions and implications cannot be shared with any party outside the EU regulatory network. While maintaining this legal role of the regulators, participatory discourse is meant to achieve agreements between regulators and stakeholders and enable regulatory decisions that are responsive and accountable to the public.

\subsection{Added Value of the IRGC Discourse Recommendations for Pharmacovigilance Engagement}

The IRGC Framework recommends different engagement discourse types for different risk scenario types. The term 'discourse' describes a formal, orderly and usually extended expression of thought on a subject and related interchanges in speech or writing [40]. While instrumental discourse discusses practical aspects of implementing risk minimisation action, a discourse of epistemology is concerned with maximising what can be known on the risk and possible actions. Furthermore, a discourse of reflective nature aims at agreeing a reasoned way forward despite uncertainty, and building resilience for coping with uncertainty and knowledge changes, while a participatory discourse is a debate for building tolerance, resolving conflicts and establishing common ground between stakeholders, and subsequently achieving agreements on risk minimisation [12,13] (see Table 3). The framework's broader vision on discourse, rather than on specific formats of engagement events, can be seen as adding value for tailoring engagement events to the environment and objectives of regulatory pharmacovigilance. This vision encourages deeper thinking about which discourse type is most suitable for creating the texture of an engagement event that may effectively obtain input from stakeholders at any specific stage of risk assessment and enable regulatory decisions that are likely to manage the given risk in the given circumstances. This focus on texture may help with selecting the appropriate engagement mechanism in given risk scenarios and fine-tune the conduct of stakeholder interactions. Recent interview research with patient organisations in Europe has already identified the need for supporting patients engaging in regulatory activities with broad awareness campaigns, sessions at patient academies, and an overall proactive approach of regulatory bodies [41]. The analysis of the iconic cases included the interactive dynamics and the dimension of texture overall, and highlighted how the respective engagements in these cases motivated certain decisions and behaviours of all involved. Among more recent PRAC engagement events is the second public hearing at EMA in 2018 regarding quinolone antibiotics [42]. The scope of this hearing was mainly for patients to detail their adverse experiences after exposure to quinolones. According to the IRGC Framework, such an epistemological discourse does not necessarily have to happen in the public domain. However, other reasons in favour of publicity in the case of quinolones could have been important for stakeholders and hence EMA. Therefore, it would be informative to review further cases of PRAC engagement for learning and systematising future engagement. This could also take into account the lessons learnt by EMA from stakeholder interactions in other risk areas that are not led by PRAC, such as $\mathrm{N}$-nitrosamine impurities of angiotensin II receptor antagonists (medicines against high blood pressure, also known as 'sartans') [43].

\subsection{Proposals for Selecting Engagement Mechanisms for Regulatory Risk Assessment and Decision Making}

Given the applicability of the IRGC Framework to pharmacovigilance, the IRGC discourse recommendations may be valuable for deriving a proposed guide for regulators, in the EU and possibly elsewhere, as support when selecting engagement mechanisms for stakeholder input to regulatory risk assessment procedures.

Depth of Engagement: Considering the above discussion, it is proposed to select the engagement mechanism that provides for a more interactive discourse with the potential for more depth of engagement whenever there is unclarity over the risk scenario type of a given safety concern. It can be further proposed to involve stakeholders more than once during a risk assessment procedure through various mechanisms and with different questions, as the risk might shift the risk scenario type during the assessment. In recent years, PRAC 
has, in some instances, already called upon patients and healthcare professional representatives several times during the same procedure, with variable levels of depth of engagement for different input needed at the respective assessment stage. For example during the valproate assessment, PRAC deployed three mechanisms, i.e. a written consultation to gather evidence on experiences with previous risk minimisation measures at the beginning of the assessment process to reduce uncertainty around the effectiveness of the measures; the public hearing described above as the appropriate engagement mechanism for situations of ambiguity due to different stakeholder perspectives; and a subsequent dedicated meeting shortly before regulatory decision making to discuss preferences and practicalities of potential future risk minimisation measures $[33,34]$. The dedicated meeting corresponded with an instrumental discourse, which is recommended by the IRGC Framework for simple risk scenarios with evidence on causality of the risk and effectiveness of existing or possible risk minimisation or precautionary measures. For risk management of medicines, an instrumental discourse on how to put measures into practice can actually be seen as necessary in all four risk scenarios. This could happen as formative research before or after a decision on regulatory action has been taken. At these time points, the discourse can focus on the design, dissemination and further aspects of the implementation of measures in healthcare for improving patient safety. For the full implementation that ensures that the measures reach patients along the clinical care pathway, further local instrumental discourse might be necessary in healthcare settings.

Breadth of Engagement: Considering the above discussion regarding the compatibility of the IRGC discourse recommendations with medicines regulation, the breadth of each engagement event should be arranged in ways that balance the needs for scope and timeliness of stakeholder input with the availability of stakeholders.

Texture of Engagement: Different from written consultations, face-to-face interactions provide for a higher degree of texture. Demonstrating trustworthiness of the regulatory body is seen by patient representatives as a major opportunity offered by a public hearing (François Houÿez, EURORDIS, personal communication, 3 March 2020). Recognising the added value of the IRGC Framework's vision on discourse, as discussed above, may facilitate shaping the necessary texture by adapting face-to-face engagement events in terms of setting, agenda, understandable language, formulation of questions, style of chairing and interactions (including needs for publicity or privacy) to the engagement mechanism available for the given regulatory procedure.

By matching the IRGC Framework with the regulatory risk assessment process and taking into account the aspects discussed above, proposals have been derived from the IRGC discourse recommendations and consolidated as a visual decision guide for regulators when selecting engagement mechanisms for different risk scenario types (see Fig. 1). This guide also reiterates the objective of the engagement discourse in terms of the stakeholder input and agreements needed for the regulatory assessment and decision making on risk management. Risk minimisation measures relate to who can use the product and how the product can be used safely, and uses a range of tools, such as the product information, pack size, legal status, educational materials, controlled access programmes, or specific sets of combined measures to manage risks of medicines in pregnancy [44]. Examples for precautionary measures are advice with precautions for use in the product information, or marketing suspension while investigations are ongoing for the product.

Determining the risk scenario of a given safety concern depends not only on the evidence for the risk from a pharmacological point of view but also on the evidence and experience with previous or possible measures for risk management, the stakeholder perspectives on the risk and the measures, and the overall context. All these determinants can differ between jurisdictions: risk factors for a certain adverse reaction can differ locally; more often, the implementability of measures will differ between healthcare systems and will require locally optimised measures and implementation; and stakeholders and their views may also differ locally. Therefore, applying the decision guide to the same medicinal product risk in different jurisdictions might not necessarily result in the same decision on engagement. It is also not the intent of the decision guide to harmonise engagement for specific medicinal product risk across various jurisdictions; the intention instead is to help address local engagement needs with a systematic approach.

\section{Conclusions}

An analysis of six iconic cases of medicine safety concerns using different mechanisms for engagement of the EU regulatory network with patients and healthcare professionals during risk assessment demonstrated that the IRGC Framework appears applicable to pharmacovigilance.

A practical, visual decision guide has therefore been derived from the IRGC Framework and tailored for pharmacovigilance purposes as a proposal for regulators when selecting mechanisms for their engagement with patients and healthcare professionals. With the decision guide, the risk scenario type of a given safety concern with its nature and extent can be determined, as well as the matching discourse type. This should support ensuring that engagement is systematic and consistent across safety concerns and regulatory procedures, and provides the texture for the most purposeful discourse, i.e. to effectively obtain the needed real-world 


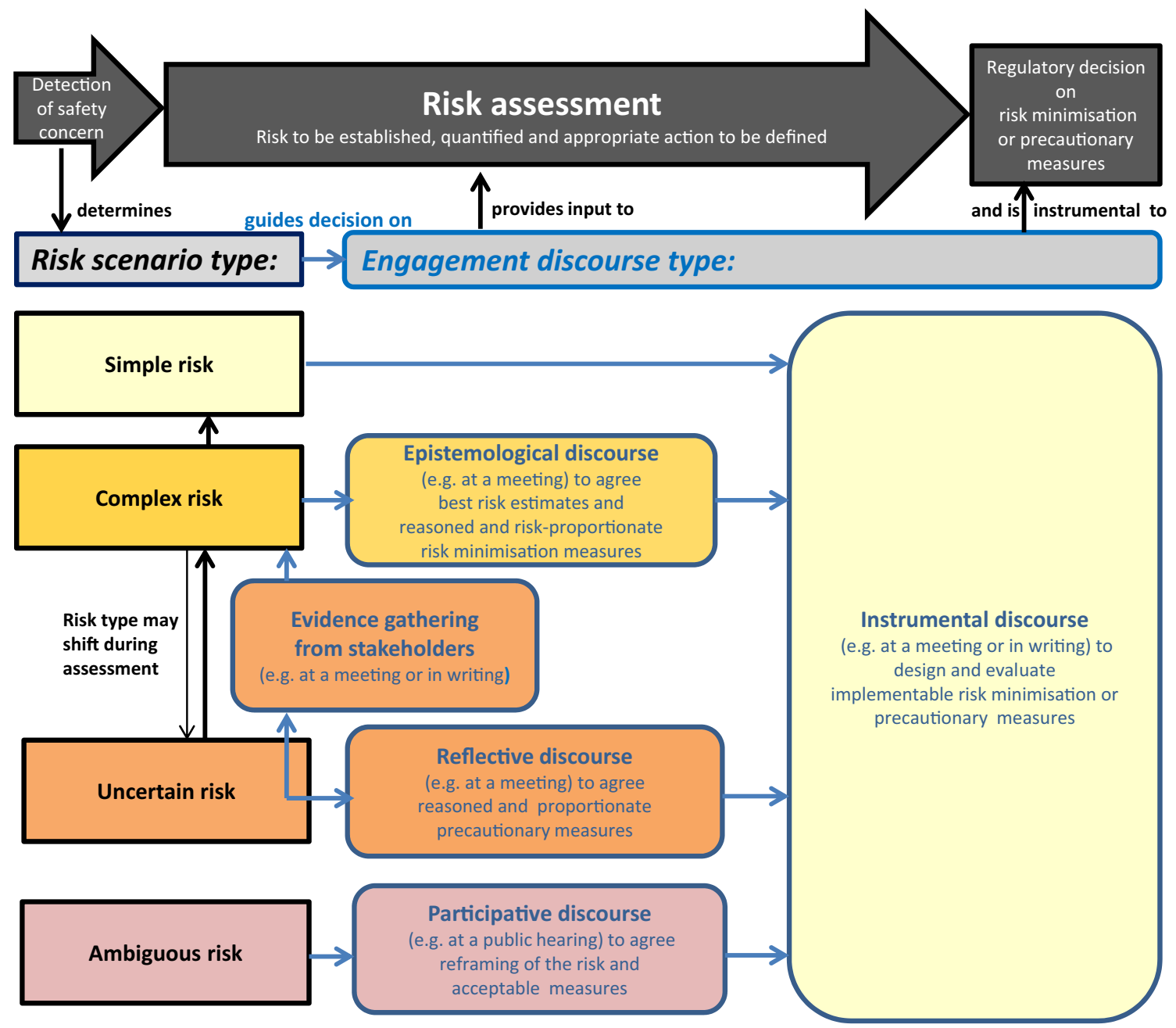

Fig. 1 Decision guide for selecting engagement mechanisms based on the discourse type required for different risk scenarios subject to regulatory risk assessment. Black frames and arrows indicate items relating to the risk scenario and the risk assessment process, while blue frames and arrows indicate items relating to the decision on the

input at the right time point for regulatory risk assessment, evaluation of risk minimisation measures and decision making. In addition, it should ensure that resources for engagement events are used in an efficient and risk proportionate manner for those risk scenarios where engagement is most needed.

Adopting specific expertise in effective discourse for pharmacovigilance matters and patient safety may facilitate strengthening regulatory engagement processes and outcomes. Expertise in discourse is suggested here as a novel competence within pharmacovigilance. The case study on valproate (referenced in Table 5) has already resulted in proposals for looking into how engagement can create common ground, a sense of shared problem ownership, joint evaluations and agreement on risk management solutions, as well discourse type for stakeholder engagement to support the risk assessment outcome. The respective discourse types recommended for each of the four risk scenario types are indicated by the matching colours of the boxes depicting the risk scenario and the engagement types

as leadership for implementation of risk minimisation or precautionary measures in healthcare.

The applicability of the IRGC Framework to pharmacovigilance has been presented to PRAC. Next, the proposed decision guide is planned to be taken forward with considering learnings from more recent engagement events, piloting it with current PRAC assessments, and subsequent refinement as a truly practical guide. Training for regulators involved in PRAC activities on how to apply the guide, along with their feedback, will be part of the iterative refinement. The decision guide are intended to be integrated into points to consider for PRAC, supporting their purposeful use of all engagement mechanisms established at EMA. These points to consider are under development as part of the PRAC Impact Strategy, and will also integrate the existing EMA 
rules of procedure on public hearings, the pharmacovigilance engagement concept (described in Sect. 1) and the Analysing Stakeholder Safety Engagement Tool (ASSET; developed through the valproate case study referenced in Table 5). The ASSET has been suggested for ensuring that stakeholder input is solicited with a view to implementability of risk minimisation measures in healthcare, because some crucial gaps in feedback to regulators from real-world healthcare and daily patient life regarding the dissemination and further implementation of risk minimisation measures have been identified. Filling these gaps is meant to contribute to improving regulatory risk minimisation measures and, ultimately, patient health.

It is important to highlight that the 'points to consider' on PRAC engagement are being developed with input from the representatives of the patient and healthcare professional communities participating in PRAC and the PRAC Interest Group on Measuring the Impact of Pharmacovigilance Activities. Subsequently, the draft 'points to consider' will be discussed with the EMA's Patients' and Consumers' Working Party (PCWP) and Healthcare Professionals' Working Party (HCPWP), to ensure that it will meet stakeholder needs, expectations and practicalities.

Although the analysis and its objectives presented in this article relate to EMA and its risk assessment committee, PRAC, the proposals are derived from a well-established international framework and the proposed practical decision guide might serve the strengthening of pharmacovigilance engagement of regulators globally.

Acknowledgements The authors thank the PRAC Interest Group on Measuring the Impact of Pharmacovigilance Activities for reviewing the study design and welcoming the results of the case analyses. The authors also thank Nathalie Bere and Viola Macolić Šarinić at EMA for reviewing this manuscript.

The views expressed in this article are the authors' personal views and may not be understood or quoted as being made on behalf of, or reflect the position of, the EMA or one of its committees or working parties.

\section{Declarations}

Funding No specific funding was received for the conduct of this study.

Conflicts of interest Priya Bahri and Antoine Pariente have no conflicts of interests to declare. The presented work emerged from reflections and reviews conducted at EMA to inform ongoing work of the PRAC Interest Group on Measuring the Impact of Pharmacovigilance Activities. Antoine Pariente declares his role as an independent expert towards the French Medicines Agency (Agence Nationale de Securite du Médicament et des Produits de Santé; ANSM) and EMA. He coordinates the DRUGS Systematised Assessment in real-liFe Environment (DRUGS-SAFE) programme funded by the ANSM, which aims at providing an integrated system allowing the concomitant monitoring of medicines use and safety in France.
Ethics approval Not applicable.

Consent to participate Not applicable.

Availability of data and material This study used the records described and referenced.

Code availability Not applicable.

Author contributions The concept for the manuscript was proposed and the study conducted by PB in consultation with the PRAC Interest Group on Measuring the Impact of Pharmacovigilance Activities. As Chair of the PRAC Interest Group, AP conducted a critical review of the study analysis and results. Both PB and AP finalised the manuscript, approved it prior to submission to Drug Safety, and approved the final version for publication.

\section{Consent to publish}

Consent to publish statements made by François Houÿez, EURORDIS, during personal conversations with the authors has been obtained.

Open Access This article is licensed under a Creative Commons Attribution-NonCommercial 4.0 International License, which permits any non-commercial use, sharing, adaptation, distribution and reproduction in any medium or format, as long as you give appropriate credit to the original author(s) and the source, provide a link to the Creative Commons licence, and indicate if changes were made. The images or other third party material in this article are included in the article's Creative Commons licence, unless indicated otherwise in a credit line to the material. If material is not included in the article's Creative Commons licence and your intended use is not permitted by statutory regulation or exceeds the permitted use, you will need to obtain permission directly from the copyright holder. To view a copy of this licence, visit http://creativecommons.org/licenses/by-nc/4.0/.

\section{References}

1. Arlett P, Straus S, Rasi G. Pharmacovigilance 2030. Clin Pharmacol Ther. 2020;107:89-91.

2. Radawski C, Morrato E, Hornbuckle K, Bahri P, Smith M, Juhaeri $\mathrm{J}$, et al. on behalf of the ISPE BRACE SIG. Benefit-risk assessment, communication and evaluation (BRACE) throughout the life cycle of therapeutic products: overall perspective and role of the pharmacoepidemiologist. Pharmacoepidemiol Drug Saf. 2015;24:1233-40.

3. Rocca E, Anjum RL. Erice call for change: utilising patient experiences to enhance the quality and safety of healthcare. Drug Saf. 2020;43:513-5.

4. Brown P, Bahri P. 'Engagement' of patients and healthcare professionals in regulatory pharmacovigilance: establishing a conceptual and methodological framework. Europ J Clin Pharmacol. 2019;75:1181-92.

5. Rowe G, Frewer L. A typology of public engagement mechanisms. Sci Technol Hum Values. 2005;30:251-90.

6. Pharmacovigilance Risk Assessment Committee (PRAC). PRAC strategy on measuring the impact of pharmacovigilance activities. London: European Medicines Agency; 2016, rev 1 2017. https:// www.ema.europa.eu/en/documents/other/prac-strategy-measu ring-impact-pharmacovigilance-activities_en.pdf. Accessed 18 Mar 2021. 
7. Teston CB, Graham SS, Baldwinson R, Li A, Swift J. Public voices in pharmaceutical deliberations: negotiating "Clinical Benefit" in the FDA's Avastin hearing. J Med Humanit. 2014;35:149-70.

8. US FDA. FDA patient-focussed drug development guidance series for enhancing the incorporation of the patient's voice in medical product development and regulatory decision making. Silver Spring, MD: US FDA; 2020. https://www.fda.gov/drugs/devel opment-approval-process-drugs/fda-patient-focused-drug-devel opment-guidance-series-enhancing-incorporation-patients-voicemedical. Accessed 18 Mar 2021.

9. US FDA. Guidance for the public, FDA Advisory Committee members, and FDA staff: the open public hearing at FDA Advisory Committee meetings. Silver Spring, MD: US FDA; 2013. https://www.fda.gov/regulatory-information/search-fda-guida nce-documents/open-public-hearing-fda-advisory-committeemeetings. Accessed 19 Oct 2020.

10. European Medicines Agency. Rules of procedure on the organisation and conduct of public hearings at the Pharmacovigilance Risk Assessment Committee (PRAC) (rev 1). Amsterdam: European Medicines Agency; 2020. https://www.ema.europa.eu/en/docum ents/regulatory-procedural-guideline/rules-procedure-organisati on-conduct-public-hearings-pharmacovigilance-risk-assessmentcommittee_en.pdf. Accessed 18 Mar 2021.

11. European Medicines Agency. Partners and networks. Amsterdam: European Medicines Agency; 2020. https://www.ema.europa.eu/ en/partners-networks. Accessed 19 Oct 2020.

12. International Risk Governance Council (IRGC). Introduction to the IRGC Risk Governance Framework (revised version). Lausanne: EPFL International Risk Governance Center; 2017.

13. Renn O, with annexes by Graham P, on behalf of the IRGC Scientific and Technical Council. White paper on risk governance: Towards an integrative approach. Geneva: International Risk Governance Council (IRGC); 2005.

14. Collins Dictionary. Iconic. https://www.collinsdictionary.com/ dictionary/english/iconic. Accessed 20 Jul 2020.

15. Merriam-Webster Dictionary. Iconic. https://www.merriam-webst er.com/dictionary/iconic. Accessed 20 Jul 2020.

16. Oxford Dictionary. Iconic. https://www.lexico.com/definition/ iconic. Accessed 20 Jul 2020.

17. European Medicines Agency. Combined hormonal contraceptives. London: European Medicines Agency; 2013. https://www.ema. europa.eu/en/medicines/human/referrals/combined-hormonalcontraceptives. Accessed 20 Jul 2020.

18. Bahri P. Impact of stakeholder consultations on risk communication and implications for pharmacoepidemiology: the case of venous thromboembolism with combined hormonal contraceptives in the EU. In: Bahri P, editor. Communicating about risks and safe use of medicines: real life and applied research. Singapore: Adis/Springer Nature; 2020. p. 106-19.

19. European Medicines Agency. Crixivan ${ }^{\circledR}$. London: European Medicines Agency; 2018. https://www.ema.europa.eu/en/medic ines/human/EPAR/crixivan. Accessed 19 Oct 2020.

20. European Medicines Agency. Viracept ${ }^{\circledR}$. London: European Medicines Agency; 2014. https://www.ema.europa.eu/en/medicines/ human/EPAR/viracept. Accessed 20 Jul 2020.

21. European Medicines Agency. Norvir ${ }^{\circledR}$. London: European Medicines Agency; 2015. https://www.ema.europa.eu/en/medicines/ human/EPAR/norvir. Accessed 19 Oct 2020.

22. European Medicines Agency. Invirase ${ }^{\circledR}$. London: EMA; 2018. https://www.ema.europa.eu/en/medicines/human/EPAR/invirase. Accessed 19 Oct 2020.

23. European Medicines Agency. Fortovase ${ }^{\circledR}$. London: European Medicines Agency; 2006. https://www.ema.europa.eu/en/medic ines/human/EPAR/fortovase. Accessed 19 Oct 2020.
24. Houÿez F. From passive to active: patients as contributors to medicinal product risk communication research. In: Bahri P, editor. Communicating about risks and safe use of medicines: real life and applied research. Singapore: Adis/Springer Nature; 2020. p. $457-80$.

25. Leonardo Alves TIM. Patient perspectives in pharmaceutical policy: information and influence in the diffusion of new medicines [PhD thesis]. Utrecht: Utrecht University; 2018.

26. Bouder F. Benefit/risk communication by the European Medicines Agency: a study of influential stakeholders' expectations and attitudes. London: European Medicines Agency; 2011. https://www. ema.europa.eu/en/documents/report/benefit/risk-communicationeuropean-medicines-agency-study-influential-stakeholders-expec tations-attitudes_en.pdf. Accessed 4 Jan 2021.

27. European Medicines Agency. Thalidomide Celgene ${ }^{\circledR}$ (previously Thalidomide Pharmion ${ }^{\circledR}$ ). Amsterdam: 2019. https://www.ema. europa.eu/en/medicines/human/EPAR/thalidomide-celgene. Accessed 27 Oct 2020.

28. Bahri P. A multilayered research framework for humanities and epidemiology of medicinal product risk communication. In: Bahri $\mathrm{P}$, editor. Communicating about risks and safe use of medicines: real life and applied research. Singapore: Adis/Springer Nature; 2020. p. 1-84.

29. European Medicines Agency. Tysabri ${ }^{\circledR}$. Amsterdam: European Medicines Agency; 2020. https://www.ema.europa.eu/en/medic ines/human/EPAR/tysabri. Accessed 27 Oct 2020.

30. European Medicines Agency. Second report on the progress of the interaction with patients' and consumers' organisations and analysis of the degree of satisfaction of patients and consumers involved in EMEA activities during 2008. London: European Medicines Agency; 2009. https://www.ema.europa.eu/en/documents/report/ second-report-progress-interaction-patients-consumers-organisati ons-analysis-degree-satisfaction/consumers-involved-europeanmedicines-agency-activities-during-2008_en.pdf. Accessed 27 Oct 2020.

31. Nixon R, Waddingham E, Mt-Isa S, Hockley K, Elmachtoub A, Gelb D, et al. Natalizumab wave 2 case study report: review of methodologies for benefit and risk assessment of medication, and risk benefit case study with a focus on testing methodology. Pharmacoepidemiological Research on Outcome of Therapeutics by a European Consortium (PROTECT); 2013. http://www.imi-protect. eu/documents/NixonetalBenefitRiskWave2CasestudyReportNatal izumabMarch2013.pdf. Accessed 6 Jan 2021.

32. European Medicines Agency. Scientific discussion (on Tysabri ${ }^{\circledR}$ ). Amsterdam: European Medicines Agency; 2020. https://www. ema.europa.eu/en/documents/scientific-discussion/tysabri-eparscientific-discussion_en.pdf. Accessed 27 Oct 2020.

33. European Medicines Agency. Valproate and related substances. London: European Medicines Agency; 2018. https://www.ema. europa.eu/en/medicines/human/referrals/valproate-related-subst ances-0. Accessed $20 \mathrm{Jul} 2020$.

34. Bahri P, Morals DR, Inoubli A, Dogné JM, Straus SMJM. Proposals for engaging patients and healthcare professionals in risk minimisation from an analysis of stakeholder input to the EU valproate assessment using the novel Analysing Stakeholder Safety Engagement Tool (ASSET). Drug Saf. 2021;44:193-209.

35. European Medicines Agency. Public hearing on valproate: first experience and lessons learnt. London: European Medicines Agency; 2017. https://www.ema.europa.eu/en/documents/report/ public-hearing-valproate-first-experience-lessons-learnt_en.pdf. Accessed 3 Jun 2020.

36. European Medicines Agency. Public stakeholder meeting: development and authorisation of safe and effective COVID-19 vaccines in the EU. Amsterdam: European Medicines Agency; Dec 2020. https://www.ema.europa.eu/en/events/public-stake 
holder-meeting-development-authorisation-safe-effective-covid19-vaccines-eu. Accessed 4 Jan 2021.

37. Regulation (EC) No 726/2004 of the European Parliament and of the Council of 31 March 2004 as amended [consolidated version]. Brussels: European Commission; 2019. https://eur-lex.europa.eu/ legal-content/EN/TXT/PDF/?uri=CELEX:02004R0726-20190 $128 \&$ from $=\mathrm{EN}$. Accessed 8 Feb 2021.

38. Møllebæk M. Rhetoric and science and technology studies. In: Bahri P, editor. Communicating about risks and safe use of medicines: real life and applied research. Singapore: Adis/Springer Nature; 2020. p. 267-84.

39. Bhasale AL, Sarpatwari A, De Bruin ML, Lexchin J, Lopert R, Bahri P, et al. Post-market safety communication for protection of public health: a comparison of regulatory policy in Australia, Canada, the European Union, and the United States. Clin Pharmacol Ther. 2020;109:1424-42.

40. Merriam-Webster Dictionary. Discourse. https://www.merriamwebster.com/dictionary/discourse. Accessed 11 Feb 2021.

41. Chinchilla K, Matos C, Hall V, van Hunsel F. Patient organizations' barriers in pharmacovigilance and strategies to stimulate their participation. Drug Saf. 2021;44:181-91.
42. European Medicines Agency. Quinolone- and fluoroquinolonecontaining medicinal products-public hearing. London: European Medicines Agency; 2018. https://www.ema.europa.eu/en/ medicines/human/referrals/quinolone-fluoroquinolone-conta ining-medicinal-products\#public-hearing-section. Accessed 5 Mar 2021.

43. European Medicines Agency. Lessons learnt from presence of $\mathrm{N}$-nitrosamine impurities in sartan medicines. Amsterdam: European Medicines Agency; 2019. https://www.ema.europa.eu/en/ documents/report/lessons-learnt-presence-n-nitrosamine-impur ities-sartan-medicines_en.pdf. Accessed 24 Jun 2021.

44. European Medicines Agency and Heads of Medicines Agencies. Guideline on good pharmacovigilance practices (EU-GVP) Module XVI: Risk minimisation measures: selection of tools and effectiveness indicators (draft rev 3). Amsterdam: European Medicines Agency; 2021. https://www.ema.europa.eu/en/human-regul atory/post-authorisation/pharmacovigilance/good-pharmacovi gilance-practices. Accessed 6 Apr 2021. 